\title{
O CRESCIMENTO ECONÔMICO NA FAIXA DE FRONTEIRA DE 2000 A 2010: O CASO DO PARANÁ
}

\section{ECONOMIC GROWTH IN THE BORDER STRIP FROM 2000 TO 2010: THE CASE OF PARANÁ}

\author{
Alberto Alves da Rocha ${ }^{1}$ \\ Camili Dal Pai ${ }^{2}$ \\ Isabela Barchet ${ }^{3}$ \\ Ricardo Rippel ${ }^{4}$
}

\section{RESUMO}

O objetivo deste artigo é analisar a distribuição espacial do desenvolvimento como o reflexo do crescimento econômico na faixa de fronteira do estado do Paraná. Para tanto, foram utilizados os dados do Índice FIRJAN de Desenvolvimento Municipal (IFDM), entre os anos de 2000 e 2010, e as categorias de Emprego e Renda, Educação e Saúde que o compõem. Foram utilizados a estatística de Moran e o índice de associação espacial local (LISA), bem como a distribuição espacial geoprocessada dos dados na região em estudo. Os resultados obtidos permitem observar a formação de agrupamentos padronizados (clusters) que se formam em respostas aos incentivos das políticas públicas e em consequência aumenta o desenvolvimento local e regional. Por outro lado, observa-se que algumas unidades geográficas apresentam dados inconsistentes com a vizinhança (outliers), ou seja, observações anormais e diferentes da vizinhança. $\mathrm{O}$ estudo da distribuição espacial dos dados na faixa de fronteira permitiu comparar a evolução do crescimento econômico promovendo o desenvolvimento no período estudado. Para esta análise, observou-se a importância de considerar os fatores socioeconômicos e culturais, bem como a localização geográfica, que permitem o desenvolvimento da região de fronteira do Paraná.

Palavras-chave: Desenvolvimento regional. Análise espacial. Índice de Moran. Geoprocessamento. Faixa de fronteira.

\begin{abstract}
This paper aims to analyze the spatial distribution of development as reflecting economic growth in the border strip the state of Paraná. Therefore, we used data from the Índice FIRJAN de Desenvolvimento Municipal (IFDM), between the years 2000 and 2010, broken down into categories of Employment and Income, Education and Health. Was used for the statistical Moran and the Index of Local Spatial Association (LISA) and the spatial distribution geoprocessada data in the study area. The results obtained allow us to observe the formation of standardized groupings (clusters) that are formed in response to the incentives of public policies and consequently increases the local and regional development. On the other hand, it is observed that some units have geographical data inconsistent with the neighborhood (outliers), or abnormal observations and different neighborhood. The spatial distribution of the data in the
\end{abstract}

\footnotetext{
${ }^{1}$ Geógrafo e mestre em Desenvolvimento Regional e Agronegócio pela Estadual do Oeste do Paraná (UNIOESTE) - Campus Toledo. Incra/PR - Rua Dr. Faivre, 1220, Curitiba - Telefone (41) 3360 6572. E-mail: albertodarocha@hotmail.com ${ }^{2}$ Universidade Estadual do Oeste do Paraná (UNIOESTE) - Campus Toledo. Doutoranda em Desenvolvimento Regional e Agronegócio - Universidade Estadual do Oeste do Paraná (UNIOESTE) - Campus Toledo - Rua da Faculdade, 645, Jd. Santa Maria, Toledo, PR - CEP: 85903-000 - Telefone (45) 3379 7053. E-mail: camili.dpp@ gmail.com

${ }^{3}$ Economista. Pesquisadora assistente em grupos de pesquisa da Universidade Estadual do Oeste do Paraná (UNIOESTE) e na Université du Québec à Chicoutimi (UQAC) discutindo temáticas relacionadas à Economia Regional e Urbana. Doutoranda em Desenvolvimento Regional e Agronegócio na Universidade Estadual do Oeste do Paraná (UNIOESTE - Campus Toledo) - Rua da Faculdade, 645, Jd. Santa Maria, Toledo, PR - CEP: 85903-000 - Telefone (45) 3379 7053. E-mail: isabelabarchet@ hotmail.com. ${ }^{4}$ Economista. Doutor em Demografia pela Universidade Estadual de Campinas (UNICAMP) e pós-doutor em Demografia pela Universidade Federal de Minas Gerais (UFMG). Professor adjunto da Universidade Estadual do Oeste do Paraná (UNIOESTE, Campus Toledo) - Rua da Faculdade, 645, Jd. Santa Maria, Toledo, PR - CEP: 85903-000 - Telefone (45) 3379 7053. E-mail: ricardo.rippel@unioeste.br
} 
region border strip allowed us to compare the evolution of economic growth by promoting development in the studied period prescribed. For this analysis, we observed the importance of considering the socioeconomic and cultural factors, well as the geographical location, that allow the development of the border region of Paraná.

Keywords: Regional development. Spatial analysis. Moran index. Geoprocessing. Border strip.

\section{Introdução}

Uma das principais características da informação geográfica é a sua localização espacial, que tem a capacidade de elaborar diversos dados interligados entre si e de forma dependente. Na sociedade e na natureza os fenômenos se inter-relacionam, se completam e criam uma dinâmica complexa. Esses fenômenos que ocorrem no espaço geram dados que criam a dinâmica da distribuição espacial, e podem ser estudados e analisados através de diversos recursos computacionais.

Para analisar o perfil socioeconômico de uma região específica, pode-se utilizar vários indicadores, entre eles está o Índice Firjan de Desenvolvimento Municipal (IFDM) que consiste em um estudo anual do Sistema FIRJAN, da Federação das Indústrias do Estado do Rio de Janeiro, criado a partir dos anos 2000 e permite acompanhar o desenvolvimento de todos os 5.565 municípios brasileiros em três grandes áreas de análise: Emprego e Renda, Educação e Saúde. Esse índice é elaborado, exclusivamente, com base em estatísticas públicas oficiais, disponibilizadas pelos ministérios do Trabalho e Emprego, Educação e Saúde (IFDM, 2012).

Para as principais áreas de desenvolvimento humano citadas, o IFDM considera participação igual, na leitura dos resultados, seja por áreas de desenvolvimento, seja por análise dos índices finais, variando entre 0 e 1 (quanto mais próximo de 1, maior será o nível de desenvolvimento da localidade), cuja classificação é dada conforme a Tabela 1.

Tabela 1 - Classificação do desenvolvimento do município conforme o IFDM

\begin{tabular}{l|l}
\hline entre 0,0 e 0,4 & baixo desenvolvimento \\
\hline entre 0,4 e 0,6 & desenvolvimento regular \\
\hline entre 0,6 e 0,8 & desenvolvimento moderado \\
\hline entre 0,8 e 1,0 & alto desenvolvimento \\
\hline
\end{tabular}

Fonte: IFDM (2012).

Nesse contexto, este artigo procura verificar o comportamento das disparidades do IFDM, entre os municípios da faixa de fronteira paranaense durante o período de 2000 a 2010. Por serem dados públicos do governo federal, os resultados obtidos podem 
apresentar algum viés, visto que nem todos os fenômenos são relatados e transformados em dados públicos.

\section{1 Área de estudo}

A área utilizada para o estudo compreende a faixa de fronteira paranaense, uma região com uma área de aproximadamente $65 \mathrm{mil} \mathrm{km}^{2}$ que abriga uma população de cerca de 1,6 milhão de habitantes, distribuídos em 139 municípios (IBGE, 2013). Conforme o PDFF (2009) esses municípios estão localizados no Arco Sul, na sub-região XV e em parte da sub-região XVI. As sub-regiões que compõem os Arcos Norte e Central e também o Arco Sul foram estabelecidas pelo PDFF conforme as diferenças das regiões quanto à base produtiva e identidade cultural.

Na sub-região XV, Portal do Paraná, a base produtiva é a exploração da pecuária e lavouras anuais com pouco uso de maquinários e tecnologia de produção, devido à fragilidade do solo da formação Arenito Caiuá; quanto a sua identidade cultural, é marcada pelas migrações intrarregionais das regiões Nordeste e Sudeste e pela presença de migrantes japoneses, que avançaram da região Norte paranaense, na busca de novas terras para o cultivo do café (PADIS, 2006).

A sub-região XVI - Vales Coloniais Sulinos é dividida em três segmentos, Noroeste do Rio Grande do Sul, Oeste de Santa Catarina e Sudoeste do Paraná, que apresentam características parecidas quanto a colonização, cultura e base produtiva, que é diversificada, produz matéria-prima das atividades de extração vegetal, florestal e silvicultura. $\mathrm{O}$ destaque está para o setor agroindustrial regional que atende os mercados nacional e internacional. Nota-se que a região do oeste paranaense se destaca economicamente, tanto na produção agropecuária, quanto no setor industrial e agroindustrial, seguida da região sudoeste paranaense.

Conforme Rippel (2005) a colonização dessa região iniciou nos anos de 1940 e alcançou seu ótimo de população nos anos 1960, com uma população composta em sua maioria de descentes de alemães e italianos, vindos do Rio Grande do Sul e ocupando as regiões oeste, sudoeste, centro ocidental e centro-sul paranaense. Em sua maioria esses colonizadores adquiriram pequenos lotes de terras com o objetivo de trabalhar em uma produção agrícola de uso da mão de obra familiar. 
Figura 1 - Mapa de localização dos municípios na faixa de fronteira paranaense e mesorregiões do Paraná

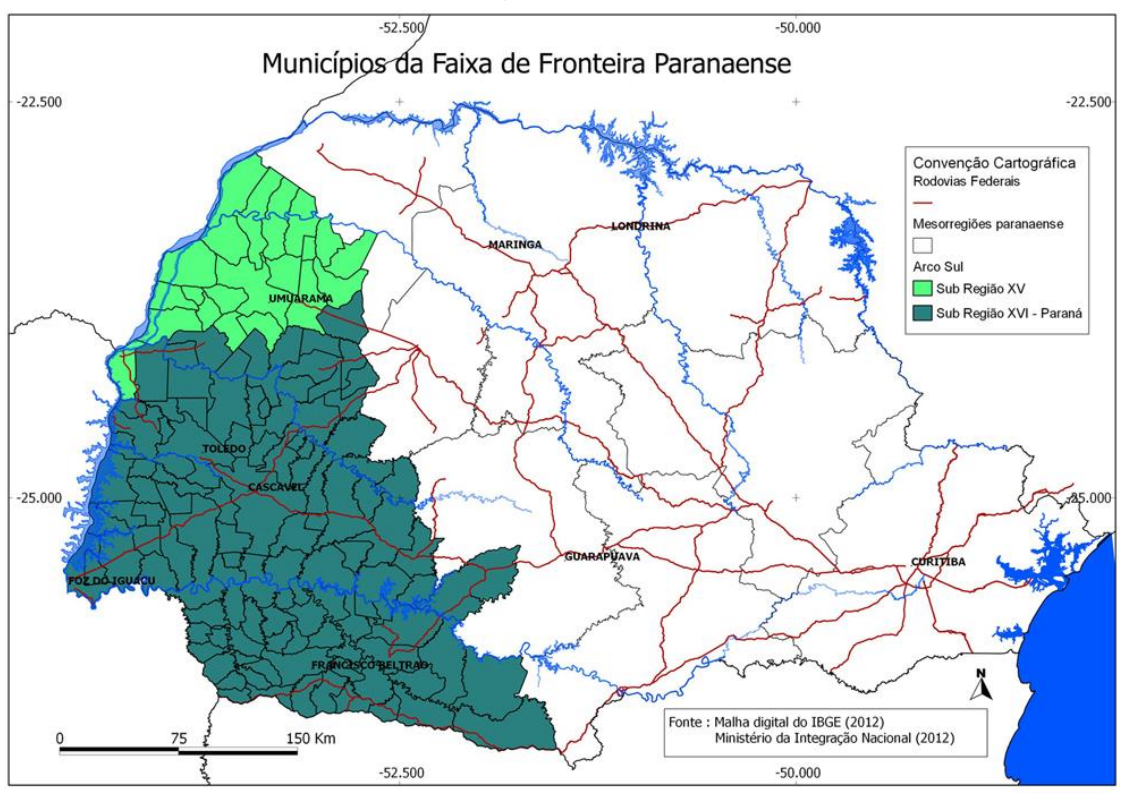

Fonte: IBGE (2012), PDFF (2009) e Ministério da Integração (2009).

Há que se destacar que formal e legalmente a faixa de fronteira brasileira é estabelecida por uma legislação nacional que determina sua dimensão em uma faixa de $150 \mathrm{~km}$, a partir da linha de fronteira. Conforme o Censo Demográfico de 2010 (IBGE, 2012), a população total da região da faixa de fronteira paranaense está acima de 2,3 milhões habitantes. Desa região mais de $80 \%$ desses municípios têm população abaixo de 20 mil habitantes, porém são considerados urbanizados conforme os critérios utilizados pelo IBGE (2012).

\section{Materiais e métodos}

Os dados do IFDM 2000 a 2010 foram obtidos através do site do sistema FIRJAN, a malha municipal vetorial foi baixada do site do Instituto Brasileiro de Geografia e Estatística (IBGE) e os dados dos limites municipais da faixa de fronteira paranaense foram elaborados durante a pesquisa, conforme as informações disponíveis no Programa de Promoção do Desenvolvimento da Faixa de Fronteira (PDFF, 2009). Para os cálculos de estatística espacial local foi utilizado o software OpenGeoDa, desenvolvido por Luc Anselin (1993) e o software IpeaGEO 1.0.5, desenvolvido pelo IPEA. São ferramentas gratuitas de análises estatísticas, voltadas especialmente a análises espaciais. Os cartogramas temáticos foram elaborados com o software QGis, multiplataforma e de uso gratuito. 


\subsection{Análise exploratória espacial de dados (AEDE)}

A análise exploratória é o uso de um conjunto de ferramentas cuja finalidade é obter um modelo estatístico inferencial que possibilite mensurar e analisar as propriedades de um fenômeno de acordo com sua localização espacial, ou seja, incorporar o espaço à análise que se deseja realizar (CÂMARA et al., 2004).

Um aspecto fundamental da análise exploratória é entender a sua dependência espacial. Segundo Anselin (1992) compreender a forma como os dados estão distribuídos no espaço é necessário para entender os indicadores da interação espacial que se apresentam através da associação ou autocorrelação espacial. E através da análise exploratória é possível observar como os valores das áreas se distribuem no espaço, e assim estimar a dimensão da dependência espacial.

Utilizou-se o teste estatístico do I de Moran Global (MORAN, 1948), para elaborar uma análise exploratória dos dados espaciais, observando a existência ou não de autocorrelação espacial nos índices do IFDM (2010) e das categorias do IFDM, Emprego e Renda, Educação e Saúde do ano de 2010.

O teste do I de Moran é uma estatística de autocorrelação espacial que representa se o espalhamento dos índices apresenta um padrão ou se mostra aleatória. Caso o espalhamento apresente um padrão espacial para as categorias do IFDM, a autocorrelação poderá ser positiva quando os valores semelhantes se apresentam próximos na distribuição espacial; ou, negativa, quando os valores se distanciam na distribuição espacial.

Conforme Marques et al. (2010, p. 3) o I de Moran Global é uma das técnicas mais utilizadas para representar correlações de variáveis. Seu valor varia de (-) 1 a (+) 1; e, quanto mais próximo de zero, menor será a existência de correlação espacial (diferenças entre vizinhos) significativa entre os valores das variáveis e o valor médio do atributo de seus vizinhos. Os valores positivos próximos à unidade geográfica indicam autocorrelação espacial positiva dos seus vizinhos, ou seja, unidades vizinhas apresentam valores similares. Já os valores negativos próximos à unidade geográfica, indicam que a autocorrelação espacial é negativa, ou seja, as unidades vizinhas apresentam valores diferentes, tanto para maior quanto para menor. 
Sobre a autocorrelação espacial, Lorena et al. (2011) explicam que o I de Moran Global fornece um único valor de medida da associação espacial para todo o conjunto de dados, o que pode ser útil quando se busca caracterizar uma região de estudo. No entanto, para identificar unidades geográficas de dependência espacial com maior precisão, Anselin (1995) recomenda que se utilizem ferramentas mais adequadas e que se apresentem resultados além do alcance dos indicadores globais; nesse caso o Índice Local de Associação Espacial (LISA).

\section{2 Índice Local de Associação Espacial (LISA)}

Para uma análise mais detalhada do processo, Camargo (2000) explica que é necessário utilizar um Índice Local de Associação Espacial (LISA), sendo que indicadores locais atribuem valores específicos para cada unidade, possibilitando identificar agrupamentos de unidades geográficas com valores de atributos semelhantes.

Neste trabalho, utilizou-se a estatística I de Moran local univariado para uma única observação, que possibilitou a realização de análises através da visualização dos dados espacialmente distribuídos e apresentados no formato de mapas e figuras.

Se o valor de I for positivo, quer dizer que a unidade geográfica está rodeada por vizinhos com valores semelhantes e apresenta padrões de associação espacial (clusters).

Se o valor de I for negativo, quer dizer que a unidade geográfica apresenta uma instabilidade local ou uma observação extrema em relação a seus valores vizinhos. Quando ocorre essas unidades são tratadas com situações atípicas (outliers), instabilidades espaciais (não estacionariedade).

Quadro 1 - Metodologia do Índice Local de Associação Espacial (LISA)

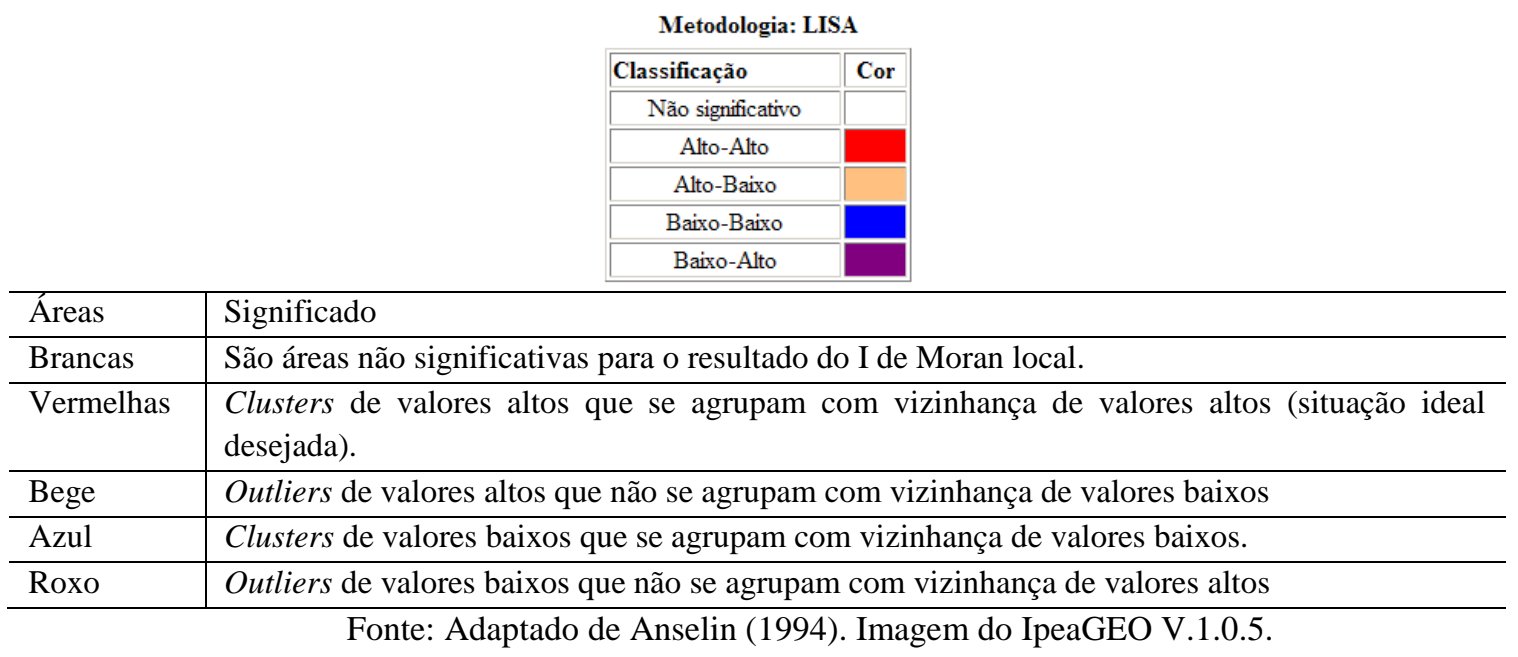


Diante do uso dessas técnicas foi possível observar e descrever a distribuição das variáveis do IFDM (2010), identificar a presença de padrões e de fenômenos atípicos em relação à distribuição e também em relação à vizinhança (ANSELIN, 1993).

De acordo com Ebdon (1985) a estatística espacial é um conjunto de técnicas de análise de padrões espaciais inferenciais utilizadas para análise e interpretação dos dados. Portanto, no caso de trabalhar com todos os dados espaciais da área de estudo, é possível postular uma hipótese nula, sendo que não é necessário observar os valores do desvio-padrão em relação à média.

\section{Resultados e discussões}

No primeiro momento foi comparada e analisada a distribuição espacial do IFDM 2000 e 2010 nos municípios da Faixa de Fronteira. Conforme a Figura 2, a distribuição espacial do IFDM de 2000 mostra que os municípios com os melhores resultados do IFDM estão localizados nas mesorregiões oeste e noroeste paranaense. De certo modo é um reflexo do processo pelo qual passava a economia nacional, com elevado desemprego e baixas taxas de crescimento, além da deterioração fiscal.

É importante ressaltar que, sem a participação do setor agroindustrial de exportação, coube ao setor agrícola e ao comércio produzir as divisas necessárias para a manutenção dos índices do IFDM de 2000, já que a região oeste é apta para a produção de soja e a região noroeste tem aptidão para produção de cana-de-açúcar e pecuária em grandes áreas, sendo que foi essa economia que movimentou a faixa de fronteira, além dos investimentos dos governos estadual e federal.

Figura 2 - Mapas da distribuição espacial do IFDM 2000 e 2010 nos municípios da faixa de fronteira paranaense

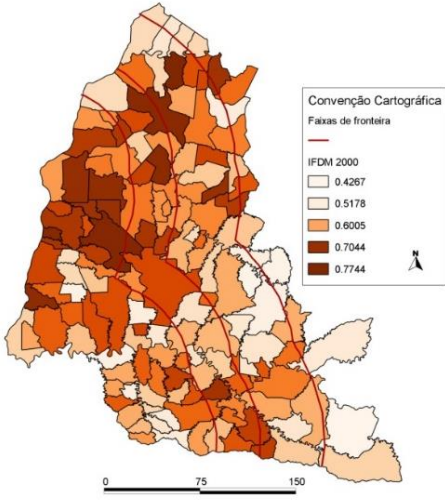

IFDM 2000

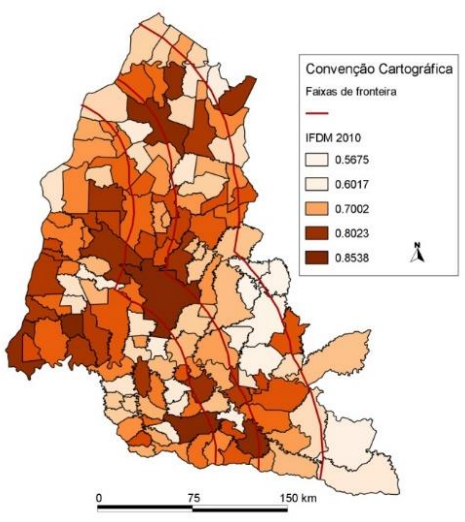

IFDM 2010

Fonte: Malha digital IBGE (2012) e IFDM (2012). 
Conforme os dados, os índices mais elevados do desenvolvimento concentramse nos municípios da mesorregião oeste paranaense, tendo Foz do Iguaçu, Toledo e Cascavel ocupando os primeiros lugares, como pode ser visto na Figura 2 da distribuição espacial do IFDM de 2010. A cidade de Foz do Iguaçu, por ser um polo turístico, tem sua economia baseada nessa atividade; Toledo e Cascavel tem a economia baseada na produção agrícola de grãos com o objetivo principal de exportar matériaprima, além de um parque agroindustrial e de indústrias metal-mecânicas. Essas atividades econômicas permitem ocupar a mão de obra local e regional, e com isso possibilita a elevação dos índices apresentados (ALVES et al., 2007).

Pode ser visto na mesorregião sudoeste que se destacam os municípios de Francisco Beltrão, Pato Branco e Dois Vizinhos por apresentarem uma economia regional com base nos setores industrial-metalúrgico, metal-mecânico, madeireiromobiliário, confecções e alimentos/bebidas. O setor de confecção apresenta-se em expansão nessa região, em que se adota um conjunto de ações, com escolas técnicas de níveis médio e superior direcionadas para formação de profissionais que atendam a demanda de mão de obra especializada (SAQUET, 2008).

Os valores elevados do IFDM 2010 na mesorregião noroeste, sub-região XV do Programa de Desenvolvimento da Faixa de Fronteira (2009), destacam os municípios Umuarama, Douradina e Rondon. Sendo Umuarama o município polo dessa região que centraliza os setores de indústria e serviços especializados; Douradina é destaque devido a seu parque industrial moveleiro que atende a demanda do Paraná, Centro-Oeste e Sudeste do Brasil, e o município de Rondon apresenta como destaque a industrialização sucroalcooleira, que ocupa a mão de obra local e dos municipios vizinhos e incrementa a economia regional. Assim, a observação do comportamento espacial das variáveis estudadas diante da conferência de mapas temáticos mostra-se importante, porém, uma análise visual da distribuição dos dados abre margens para erros.

Torna-se, assim, necessária a verificação de tendências gerais da distribuição e da formação de agrupamentos dos dados, com o uso de testes estatísticos. No segundo momento se realizou uma análise exploratória sobre o conjunto de dados, feita com base no Índice Global de Moran aplicada em separado para cada variável: IFDM, Emprego e Renda, Educação e Saúde; dos anos de 2000 e 2010 (Tabela 2), respectivamente, cujo objetivo foi avaliar a dependência espacial de cada uma. Percebe-se que o Índice Global 
de Moran (I) das variáveis IFDM 2000 (I = 0.269417) e IFDM 2010 (I =0.223793) é positivo, indicando existir uma autocorrelação espacial positiva, porém frágil por apresentar valores baixos e próximos de zero, ou seja, não significa que em todos os casos a autocorrelação seja positiva e que o valor do IFDM seja semelhante aos valores dos seus vizinhos. Para a variável IFDM Educação 2000 ( $\mathrm{I}=0.314009)$ e IFDM Educação 2010 ( $\mathrm{I}=0.176413$ ), apesar de indicar autocorrelação positiva, houve uma redução no I, ou seja, diminuiu a correlação da variável IFDM Educação 2010 com a sua vizinhança. Na análise da variável IFDM Emprego e Renda 2000 (I = -0.05241) e IFDM Emprego e Renda 2010 ( $\mathrm{I}=0.080135)$, percebe-se que a variável deixou de ser negativa no ano de 2000 e atribuída à competência da gestão pública local em gerar fazer gerar empregos. Quanto ao resultado positivo no ano de 2010, entende-se que os resultados positivos de um município interferem no resultado da vizinhança, numa situação de transbordamento e resposta das políticas de desenvolvimento regional. Para o resultado de I da variável IFDM Saúde 2000 (I = -0.08060) e IFDM Saúde 2010 (I = 0.00852), ambos os valores são negativos e indicam autocorrelação negativa, ou seja, o valor do IFDM Saúde de um município está pouco correlacionado no espaço com o valor médio do IFDM Saúde nos seus municípios vizinhos. Visto que os indicadores que compõem o IFDM Saúde estão relacionados à demanda de verbas das políticas públicas dos governos estadual e federal, tem na administração local o diferencial entre a vizinhança.

Tabela 2 - Resultados do teste de autocorrelação espacial do IFDM 2010

\begin{tabular}{lcr}
\hline Variável & $\begin{array}{l}\text { Índice de } \\
\text { Global }\end{array}$ & Moran \\
\hline IFDM 2000 & 0.269417 \\
IFDM 2010 & 0.223793 \\
Educação 2000 & 0.314009 \\
Educação 2010 & 0.176413 \\
Emprego e Renda 2000 & -0.05241 \\
Emprego e Renda 2010 & 0.080135 \\
Saúde 2000 & -0.08060 \\
Saúde 2010 & -0.00852 \\
\hline
\end{tabular}

Fonte: IFDM (2012).

Como os dados do IFDM se apresentam formatados por municípios, optou-se por executar uma análise espacial usando os softwares IpeaGEO e OpenGeoDa, conforme descritos anteriormente, através das variáveis do IFDM 2000 e 2010 e suas três categorias. O objetivo foi identificar quais municípios apresentavam formação de clusters ou outliers e a intensidade da correlação espacial, em nível local, nessa região. 
Os resultados permitiram observar e apontar quais são as regiões com maior predominância de clusters, em relação às demais regiões, bem como avaliar se a distribuição desse delito é aleatória na região de interesse. Para analisar as diferenças locais de IFDM nos anos 2000 e 2010, na ocorrência de clusters e/ou de outliers, foram confeccionados box map das categorias em IFDM, Educação, Emprego e Renda e Saúde, conforme pode ser visto na Figura 2, que faz a distinção coroplética e nominal entre os clusters e outliers expostos após a aplicação da análise estatística espacial do I de Moran.

Figura 3 - Distribuição do I de Moran Local de autocorrelação LISA para IFDM

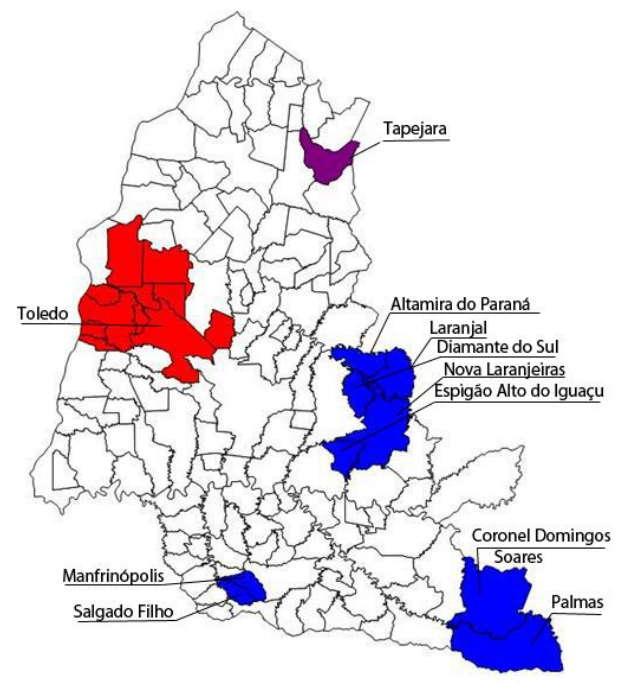

IFDM 2000

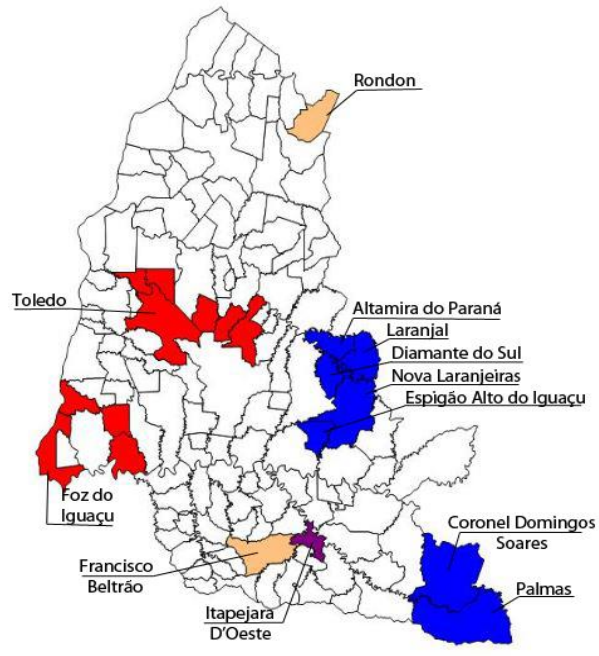

IFDM 2010

Fonte: IBGE (2012), IFDM (2012).

Nos resultados do cálculo do LISA para o IFDM 2000 e 2010 (Figura 2), nota-se que no box map do ano 2000 há a formação de quatro clusters, um Alto-Alto na microrregião de Toledo, refletindo o que é apresentado na Figura 1, no mapa de distribuição espacial do IFDM 2000. Os outros três clusters são do tipo Baixo-Baixo; o primeiro é formado pelos municípios de Altamira do Paraná, Laranjal, Diamante do Sul, Nova Laranjeiras e Espigão Alto do Iguaçu; o segundo, pelos municípios de Coronel Domingos Soares e Palmas, e o terceiro, Manfrinópolis e Salgado Filho. Os valores atribuídos a esses municípios conforme a metodologia do IFDM (2012) os classifica em desenvolvimento regular. O município de Tapejara representa um outlier do tipo BaixoAlto, que no ano de 2000, em detrimento de sua economia, projetava a relevância da economia em crescimento do município de Rondon. Esse cenário pode ser observado no resultado de 2010, em que há a formação de um outlier do tipo Alto-Baixo representado pelo município de Rondon, que faz interface fronteiriça a sul com o município de 
Tapejara. Os valores atribuídos a esses municípios, conforme a metodologia do IFDM (2012), classifica Tapejara em desenvolvimento regular nos anos 2000 e 2010, o município de Rondon ascendeu da categoria de desenvolvimento regular em 2000 para de alto desenvolvimento em 2010.

No box map de 2010, observa-se que se manteve o cluster do tipo Alto-Alto de Toledo com algumas mudanças na quantidade dos municípios, e que surgiu um novo cluster Alto-Alto em torno da microrregião de Foz do Iguaçu, cuja economia está relacionada à prestação de serviço no setor turismo. Mantêm-se dois clusters BaixoBaixo nos municípios de Nova Laranjeiras e Palmas. A presença de dois outliers, ambos do tipo Alto-Baixo, na Figura 2, em Rondon e Francisco Beltrão, e o terceiro do tipo Baixo-Alto em Itapejara D'Oeste, fazendo uma interface fronteiriça com Francisco Beltrão, descreve o contraste da mesorregião sudoeste, onde alguns municípios conseguiram aproveitar as oportunidades e estabeleceram um crescimento econômico que gerou o desenvolvimento exposto. Por outro lado, nem todos os municipios dessa mesorregião alcançaram esse crescimento, o que pode significar que não há um espraiamento do crescimento econômico que possa provocar o desenvolvimento na vizinhança.

Por outro lado, no box map da categoria IFDM Educação para o ano de 2000, Figura 6, há a presença de dois clusters do tipo Alto-Alto nos municípios de Toledo e no entorno do município de Tapira. Visto que a categoria IFDM representa tanto a oferta como a qualidade da educação infantil e do ensino fundamental nos municípios, são uma competência do governo municipal a manutenção e a qualidade nos primeiros anos do ensino. Em um comparativo, adiante, a Figura 6 mostra que a concentração da melhor oferta do ensino está na microrregião do oeste paranaense e que os municípios das mesorregiões centro-ocidental e centro-sul não alcançaram dados satisfatórios, o que pode ser atribuído à baixa arrecadação municipal de impostos e à dependência do repasse do Fundo de Participação dos Municípios (FPM), que é feito pela União. 
Figura 4 - Distribuição do I de Moran Local de autocorrelação LISA para a categoria

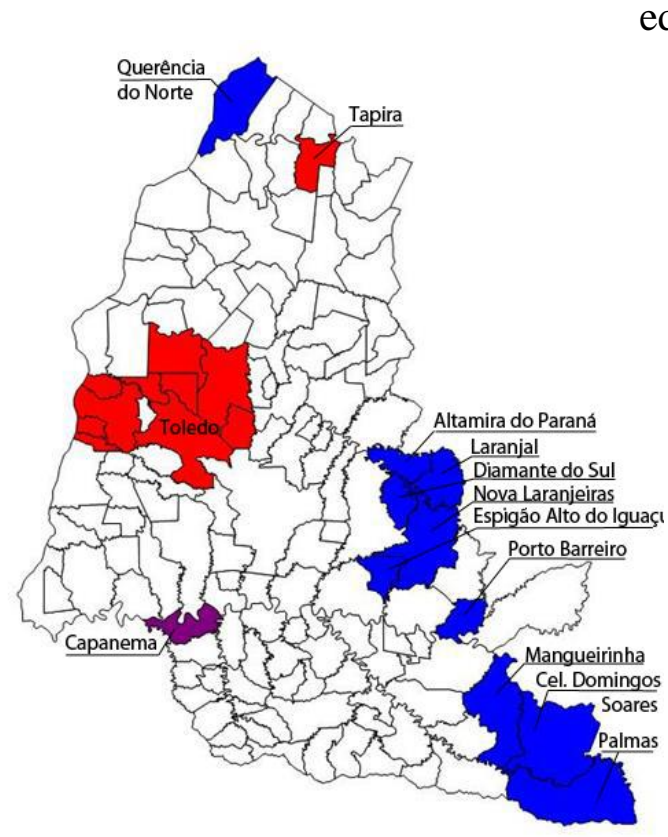

Categoria IFDM Educação 2000 educação

Fonte: IBGE (2012), IFDM (2012).

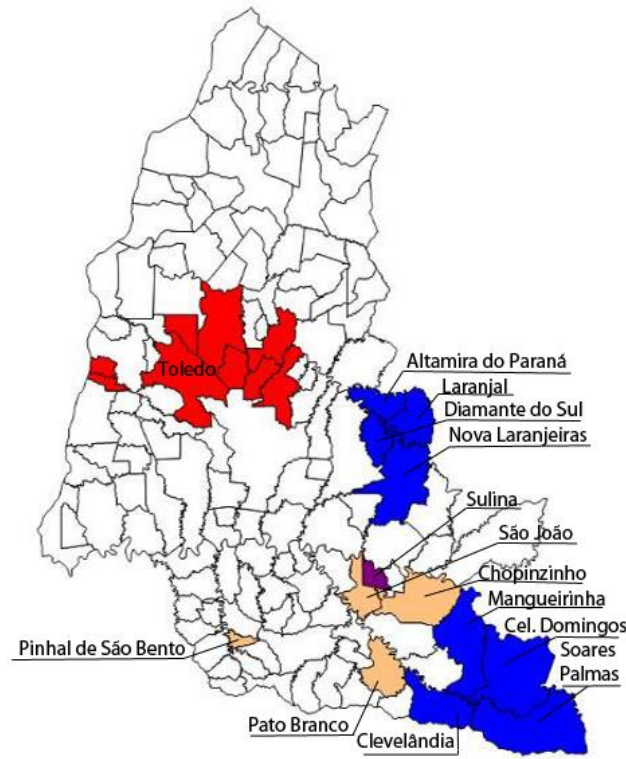

Categoria IFDM Educação 2010

Verificou-se a presença de quatro clusters do tipo Baixo-Baixo, sendo o primeiro formado pelos municípios de Altamira do Paraná, Laranjal, Diamante do Sul, Nova Laranjeiras e Espigão Alto do Iguaçu; o segundo, pelos municípios de Coronel Domingos Soares, Mangueirinha e Palmas; o terceiro, pelo município de Porto Barreiro e o quarto, pelo município de Querência do Norte.

O município de Capanema representa um outlier do tipo Baixo-Alto. Nota-se que a distribuição espacial dos clusters do tipo Baixo-Baixo abrange os municípios que foram classificados com desenvolvimento regular, conforme a metodologia do IFDM (2012). Para o ano de 2010 os clusters mantiveram-se do tipo Alto-Alto no município de Toledo e do tipo Baixo-Baixo nos municípios de Nova Laranjeiras e Palmas, com acréscimo ou redução no número de unidades geográficas. Percebe-se a presença de cinco outliers, sendo três do tipo Alto-Baixo, nos municípios de Pinhal de São Bento, Chopinzinho e Pato Branco. O município de Sulina representa o quarto outlier do tipo Baixo-Alto, fazendo interface fronteiriça com o município de São João, que é um outlier do tipo Alto-Baixo. Verificou-se que os municípios que apresentam clusters do tipo Alto-Alto foram classificados pela metodologia do IFDM (2012) com alto desenvolvimento. Aqueles que apresentam clusters do tipo Baixo-Baixo foram classificados entre baixo e desenvolvimento regular. Os municípios que apresentaram outliers Alto-Baixo foram classificados com desenvolvimento regular e moderado. Para 
os que apresentam outliers Baixo-Alto foram classificados com desenvolvimento moderado.

Figura 5 - Distribuição do I de Moran Local de autocorrelação LISA para a categoria Emprego e Renda

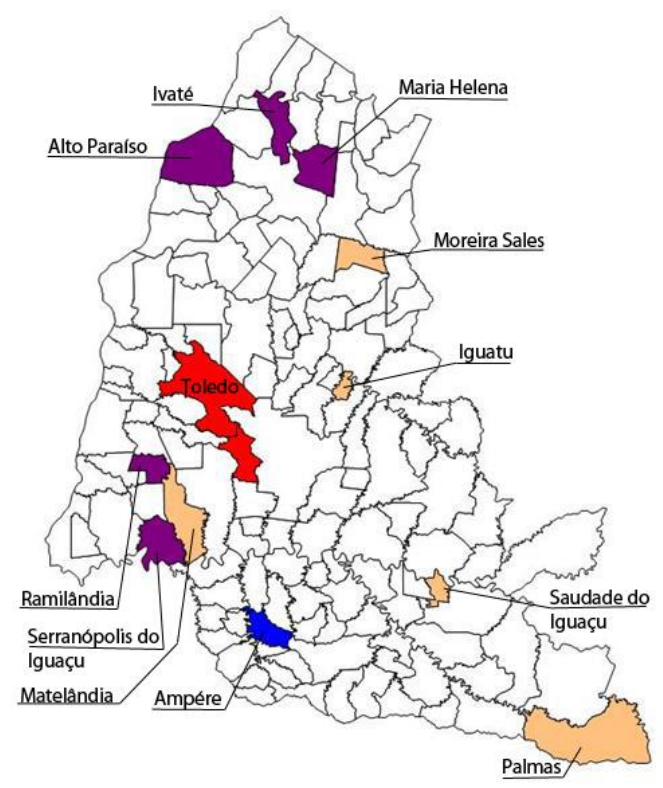

Categoria IFDM Emprego e Renda - 2000

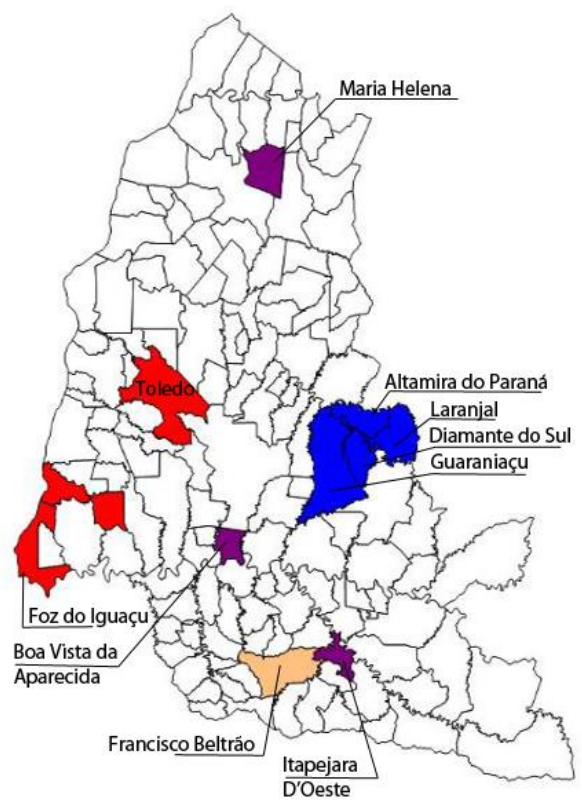

Categoria IFDM Emprego e Renda - 2010

Fonte: IBGE (2012), IFDM (2012).

Por meio dos dados é possível perceber que as regiões com os melhores resultados em gerar emprego e renda também são os que apresentaram os melhores resultados no IFDM 2010. Em comparação entre os dados de 2000 e de 2010, aproximadamente $33 \%$ dos municípios decresceram nos resultados de geração de emprego e renda. O destaque é para Xambrê, que, em 2000, apresentou o IFDM da categoria Emprego e Renda de 0,7112 ponto e, em 2010, apresentou 0,3302 ponto, saindo da classificação de município em desenvolvimento moderado para baixo desenvolvimento, o que representa um decréscimo de aproximadamente $-53 \%$.

Por outro lado, 67\% dos municípios apresentaram evolução nos resultados na categoria Emprego e Renda. O destaque foi Foz do Iguaçu que, em 2000, apresentou 0,4029 ponto e, em 2010, 0,8158 ponto, passando de desenvolvimento regular, próximo de baixo desenvolvimento pela pontuação, para alto desenvolvimento, o que equivale a um aumento de mais de $100 \%$.

Outro aumento elevado da porcentagem foi do município de Serranópolis do Iguaçu com resultado acima de $180 \%$. Porém, sobre pontuação baixa, saiu de 0,1584 
ponto em 2000 para 0,4506 ponto em 2010, elevando-se da classificação de baixo desenvolvimento para desenvolvimento regular.

Mediante esse panorama geral, vê-se que o cluster do tipo Baixo-Baixo formado pelos municípios Altamira do Paraná, Laranjal, Diamante do Sul e Guaraniaçu reflete os dados da categoria Emprego e Renda. Estes são municípios com pontuações próximas e não apresentaram crescimento nessa categoria entre 2000 e 2010.

A formação dos outliers no cartograma de 2010 (Figura 8) mostra que os municípios de Maria Helena, Boa Vista da Aparecida e Itapejara d'Oeste apresentaram crescimento do IFDM 2010, mesmo que com resultados pouco perceptíveis. No entanto, é possível verificar que a categoria Emprego e Renda 2010 não contribuiu para esse crescimento e, em 2010, essa categoria até reduziu o crescimento, ou seja, a quantidade de emprego formal em 2000 era maior que a quantidade de emprego formal em 2010. Nos municípios de Alto Paraíso, Ivaté, Ramilândia e Serranópolis do Iguaçu, que apresentaram outliers Baixo-Alto em 2000, a mudança que ocorreu em 2010 se deve ao setor responsável pela maior geração de empregos que foi a administração pública direta e indireta e o comércio varejista. A mudança da situação do outlier Baixo-Alto de Serranópolis do Iguaçu mudou a situação do outlier Alto-Baixo de Matelândia pelo fator vizinhança.

Figura 6 - Distribuição do I de Moran Local de autocorrelação LISA para a categoria Saúde

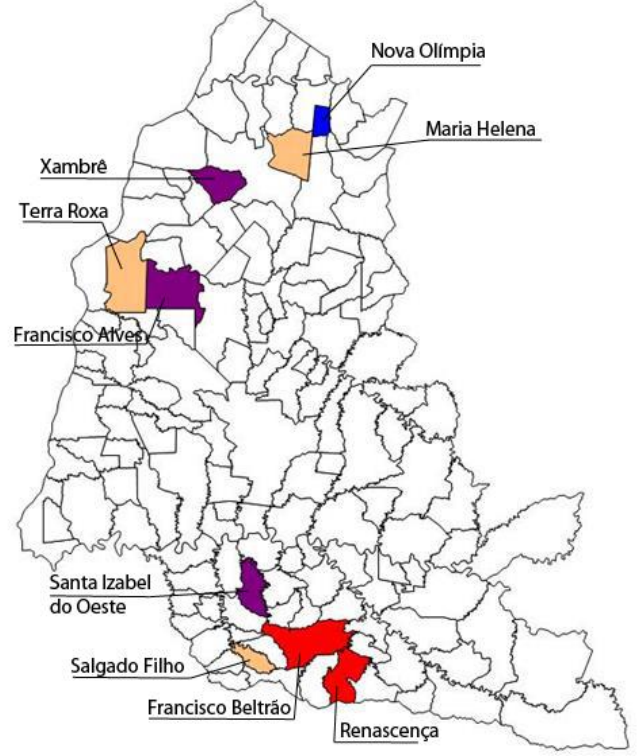

Categoria IFDM Saúde 2000

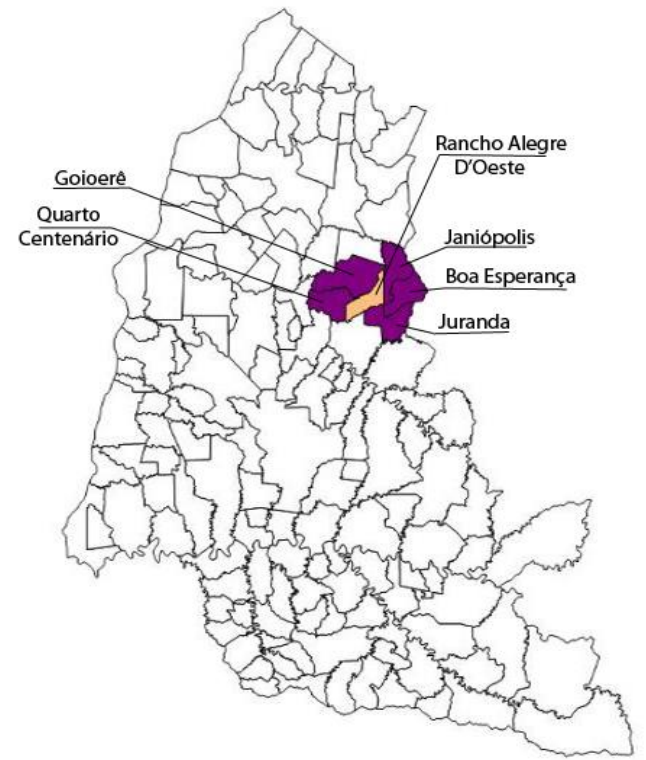

Categoria IFDM Saúde 2010

Fonte: IBGE (2012), IFDM (2012). 
$\mathrm{Na}$ análise do cartograma da categoria IFDM Saúde 2010, é possível observar que a maioria das unidades geográficas são áreas não significativas para o I de Moran, visto que esses municípios se apresentam acima de 0,8000 ponto na classificação, em alto desenvolvimento. A presença de dois outliers positivos, o primeiro do tipo BaixoAlto, se dá por Quarto Centenário, Goioere, Janiópolis, Boa Esperança e Juranda. O segundo, do tipo Baixo-Alto, que está na interface deste, é o município de Rancho Alegre d'Oeste.

A existência dos outliers está na dimensão dos dados, sendo que Rancho Alegre d'Oeste alcançou o valor máximo estabelecido, 1 ponto, e os demais municípios apresentaram mais de 0,9000 ponto na classificação da categoria IFDM Saúde 2010. Devido à formação dos outliers positivos em interface e por vizinhança, nesse caso, e por Rancho Alegre d'Oeste ter alcançado o valor máximo, a eliminação dos outliers se dá se esse município reduzir a sua pontuação ou se os demais alcançarem o valor máximo, o que causaria um espraiamento dos outliers sobre os demais municípios da vizinhança que não alcançaram o resultado máximo, mas que estão próximos disso.

\section{Considerações finais}

A análise de uma região através do Índice de Moral Local, usando os dados do IFDM e suas categorias, possibilitou ampliar a compreensão do desenvolvimento na faixa de fronteira paranaense e suas regiões que apresentam características distintas. Indicando possíveis áreas em desenvolvimento e outras menos desenvolvidas, conforme as variáveis estudadas nessa região. Este trabalho possibilitou ver as formações de desenvolvimento nessas áreas, o que serviu para entender os motivos da correlação dos municípios e o resultado dessas correlações na regionalização da região, bem como em relação ao estado.

Assim, nossa análise permite apontar que o oeste paranaense apresenta os maiores índices de correlação do tipo Alto-Alto entre seus municípios, significando que o desenvolvimento dos municípios polo dessa região provocou o espraimamento do desenvolvimento entre os municipios vizinhos. Em contrapartida, a mesorregião centrosul apresentou os maiores índices de correlação do tipo Baixo-Baixo, demonstrando agrupamentos regionais bem distintos entre seus municípios, mostrando que para alcançar um desenvolvimento regional equitativo é necessária a participação dos 
poderes públicos estadual e federal, implementando políticas de crescimento e de desenvolvimento direcionadas para essas localidades, de forma a envolver todas as partes interessadas.

Os resultados mostraram que a faixa de fronteira apresenta diferenças muito grandes e cada município tem suas singularidades. Essa região não pode ser tratada como uma região homogênea e tão pouco balizada pela média, pois apresenta municípios altamente desenvolvidos e outros que ainda estão em baixo estágio de desenvolvimento. Constatou-se que os municípios que apresentaram menores índices de desenvolvimento têm sua economia baseada no setor primário, população abaixo de 20 mil habitantes, dificuldades para proporcionar bem-estar para seus habitantes e falta de investimentos em infraestrutura básica de saúde, revestimento de ruas, iluminação pública e atendimentos médicos e hospitalar.

Entendemos que isso se deve ao processo desencadeado a partir dos anos 1970 para o desenvolvimento da faixa de fronteira, estabelecendo-se como uma economia voltada para a exportação agropecuária, produção de equipamentos e bens de consumo em escala nacional e mundial. Assim, muitos municípios da faixa de fronteira ainda não conseguiram vencer a barreira de serem somente produtores de matéria-prima e não investiram em um parque industrial capaz de gerar empregos, impostos e diminuir a dependência financeira dos repasses de verbas dos governos estadual e federal.

\section{REFERÊNCIAS}

ALVES, L. R.; FERRERA LIMA, J.; PIFFER, M.; RIPPEL, R. O continuum, a localização do emprego e a configuração espacial do oeste do Paraná. HeerA. Revista de História Econômica e Economia Regional Aplicada, v. 2, n. 2, 2007.

ANSELIN, L. Spatial Data Analysis with GIS: AnIntroduction to Application in the $\begin{array}{llll}\text { Social } & \text { Sciences. } & \text { Disponível }\end{array}$ <http://www.ncgia.ucsb.edu/Publications/Tech_Reports/92/92-10.PDF>. Acesso em: 12 jan. 2013.

ANSELIN, L. Exploratory spatial data analysis and geographic information systems. In: Workshop on New Tools for Spatial analysis. Lisboa: ISEGI, 1993. p. 18-20. 
ANSELIN, L. Local Indicators of Spatial Association-LISA. Geographical Analysis, v. 27, n. 2, p. 93-115, 1995.

ANSELIN, L.; SYABRI, I.; KHO, Y. GeoDa: An Introduction to Spatial Data Analysis. Spatial Analysis Laboratory. Department of Agricultural and Consumer Economics University of Illinois, Urbana-Champaign Urbana, 2004.

CÂMARA, G. et al. Análise espacial e geoprocessamento. In: DRUCK, S.; CARVAlho, M. S.; CÂMARA, G.; MONTEIRO, A. V. M. (Eds.). Análise espacial de dados geográficos. Brasília: EMBRAPA, 2004.

CAMARGO, E. C. G. Análise Espacial de Dados Geográficos. Laboratório. Módulo: Análise de Padrões de Áreas. Lab2_APA.doc. 2000. Disponível em: <http://www.dpi.inpe.br/cursos/ser431/labs/Lab_Areas_Spr.pdf>. Acesso em: 12 jan. 2013.

COLLEGE OF LIBERAL ARTS AND SCIENCES. GeoDa Center for Geospatial Analysis and Computation. OpenGeoDa. Disponível em: < https://geodacenter.asu.edu/ogeoda>. Acesso em: 15 mar. 2013.

EBDON, D. Statistics in Geography. Londres: Blackwell, 1985.

IBGE. Instituto Brasileiro de Geografia e Estatística. Malha digital. Disponível em: <http://www.ibge.gov.br>. Acesso em: 12 maio 2013.

IPEA. Instituto de Pesquisa Econômica Aplicada. IpeaGEO. Assessoria de Métodos Quantitativos da Diretoria de Estudos e Políticas Regionais, Urbanos e Ambientais do Ipea. Disponível em: <http://www.ipea.gov.br/ipeageo/>. Acesso em: 12 jan. 2013.

IFDM. Índice Firjan de Desenvolvimento Municipal. Versão Completa. Disponível em: < http://www.firjan.org.br/ifdm/downloads/>. Acesso em: 5 maio 2013.

LORENA, R. B.; BERGAMASCHI, R. B.; LEITE, G. R. Análise Exploratória Espacial do Índice de Desenvolvimento Humano Municipal do Estado do Espírito Santo. In: XV Simpósio Brasileiro de Sensoriamento Remoto - SBSR, Curitiba, PR, 30 de abril a 05 de maio de 2011. Anais XV Simpósio Brasileiro de Sensoriamento Remoto, São Paulo: INPE, 2011. p. 4776-4782. 
MORAN, P. A. P. The interpretation of statistical maps. Journal of the Royal Statistical Society, B10, p. 243-251, 1948.

NUNES, F. G. Análise exploratória espacial de indicadores de desenvolvimento socioambiental das regiões de planejamento do norte e nordeste goiano. Ateliê Geográfico, v. 7, n. 1, p. 237-259, 2013.

PADIS, P. C. Formação de uma economia periférica. O caso do Paraná. 2.ed. Curitiba: IPARDES, 2006.

QUANTUMGIS. QGis Brasil. QGis. Disponível em: <http://qgisbrasil.org/>. Acesso em: 20 mar. 2013.

RIPPEL, R. Migração e desenvolvimento econômico no oeste do estado do Paraná: uma análise de 1950 a 2000. 2005. Tese (Doutorado em Demografia). Instituto de Filosofia e Ciências Humanas, Universidade Estadual de Campinas, Campinas, 2005.

SAQUET, D. B. A expansão da indústria de confecções no sudoeste do Paraná. Geosul, v. 23, n. 46, p. 55-78, 2008.

Recebimento dos originais: 20/05/2014 Aceitação para publicação: 25/08/2014 